\title{
Maximizing the Mental and the Physical: Pre- Performance Routines of Elite Squash Athletes
}

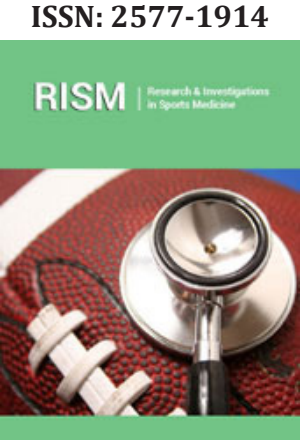

*Corresponding author: JC Muran, Gordon F Derner School of Psychology, Adelphi University, 158 Cambridge Avenue, Garden City, NY 11530, USA

Submission: 侮 January 06, 2020

Published: 㸃January 24, 2020

Volume 6 - Issue 1

How to cite this article: AC Muran, JC Muran, SK Sobhy, BS Gorman. Maximizing the Mental and the Physical: PrePerformance Routines of Elite Squash Athletes. Res Inves Sports Med, 5(5): 469473.

DOI: $10.31031 /$ RISM.2020.06.000628

Copyright@ JC Muran, This article is distributed under the terms of the Creative Commons Attribution 4.0 International License, which permits unrestricted use and redistribution provided that the original author and source are credited.

\author{
AC Muran'1, JC Muran*2,3, SK Sobhy4 and BS Gorman² \\ ${ }^{1}$ College of Arts \& Sciences, Cornell University, USA; School of Clinical Medicine, University of \\ Cambridge, UK
}

${ }^{2}$ Gordon F Derner School of Psychology, Adelphi University, USA

${ }^{3}$ Department of Psychiatry, Icahn School of Medicine at Mount Sinai, USA

${ }^{4}$ Harvard College, Harvard University, USA

\begin{abstract}
Objective: The objective of the study was to provide a foundational understanding of mental and physical pre-performance routines of elite squash athletes.

Method: The sample included 18 squash professionals who were ranked in the top 10 in the world, balanced by gender, generation, and nationality. Each participated in a semi-structured interview that addressed pre-tournament and pre-match routines, plus questions about pre-planned strategies applied in-match and general dispositional variables.
\end{abstract}

Result: Descriptive qualitative analyses supported by a web-based text analysis tool yielded a number of common and unique routines with regard to training protocol, sleep hygiene, diet regimen, mental work, tactical preparation, and physical warmup. The importance of adaptability and acceptance of pressure were also suggested. One difference by nationality was noted: religious faith.

Conclusion: There was a good deal of commonality, but also examples of individuality, in PPRs for professional squash athletes.

Keywords: Pre-performance routines; Athletic competition; Physical; Mental; Emotion regulation; Psychological resilience

\section{Introduction}

Over the past 40 years, there has been a proliferation of interest in performance science, specifically towards developing and evaluating strategies to promote peak performance (see $[1,2]$ for popular translations from the empirical literature). One such strategy that has received great attention is the use of a pre-performance routine (PPR). PPRs have long existed as part of an athlete's regimen [3]. These have been defined as an ordered collection or "sequence of task-relevant thoughts and actions which an athlete engages in systematically prior to his or her performance of a specific sports skill”([4], p. 177; see also [3,5]).

As suggested, PPRs include covert (mental or psychological) and overt (physical or behavioral) elements -procedures to promote optimal mental and physical states of readiness $[3,6]$. The former typically concerns strategies to focus attention, to maximize concentration, and to regulate anxiety. The latter concerns strategies to prepare the body's condition to perform, including optimal arousal and behavioral consistency. The essential characteristics of PPRs have been defined by the following steps: readying, imaging, focusing, executing, and evaluating [7]. The literature also makes a distinction between pre-shot routines implemented for closed skills (such as a golf shot, basketball free throw, soccer penalty kick) and pre-competitive routines used for open skills (such as a match, game, or competitive event). These are distinguished from rituals that lack instrumental purpose (formal repetitive symbolic actions, otherwise described as superstitious behaviors [8]

The empirical research has demonstrated that PPRs have a significant positive impact on performance, one of the most robust interventions shown to improve execution quality 
and reduce choking behavior (see [6,9] for reviews). Their effect includes maximizing attentional control (that is, increasing focus on task-relevant cues, decreasing focus on the negative, and reducing distractibility). Consistent and appropriate duration of application and integration of mental and physical elements are particularly important to their effect. They have also been shown to regulate anxiety and promote self-control and resilience (for example, $[10,11])$. Individualizing PPRs, tailoring the nature of the routine to the person, has been highlighted as an important aspect to their effect $[3,6]$.

Most of the research on PPRs has concentrated on closed skills, and relatively little on such skills in racquet sports, the serve in tennis being the example [6,9]. This paper presents a study of PPRs (mental and physical strategies) applied by elite squash athletes with an open skill orientation in order to define common and unique pre-tournament and pre-match preparations -with some attention to pre-planned in-match routines and general dispositional variables. It is the first on this racquet sport and includes a balanced sample of male and female athletes across nationalities who achieved a top 10 world ranking (the majority former \#1s) - some active, some retired. The method includes a semi-structured interview developed for this study; responses to which were submitted to a descriptive qualitative analysis with support from a web-based text analysis tool.

\section{Method}

\section{Participants}

Eighteen squash athletes were selected for participation using a chain-sampling method. Inclusion criteria were: (1) ranked in the top 10 at some point in career, (2) gender (female: male) evenly distributed, (3) nationality reasonably distributed, and (4) generation (active: retired) also reasonably distributed. . All provided informed consent for analysis of their interview data, following Institutional Review Board standards. There were 12 (67\%) participants who were ranked \#1 in the world. Of the nine women, average age was 32 (range 21-42), and five were still active professionals; their nationalities were: three UK, two Egypt, one US, one South African, one Australia, and one South Asia. Of the nine men, average age was 38 (range 27-46), and four were still active; their nationalities were: three Egypt, two UK, two EU, one Australia, and one South Asia. Interviews were evenly conducted by two topranked US collegiate squash athletes: one female, one male, both age 22 and of US nationality.

\section{Interview}

All participants completed a semi-structured interview about their respective preparatory routines (mental and physical) before (1) tournaments (1-2 weeks in advance) and (2) matches (day before through day of), plus (3) pre-planned in-match routines and (4) general dispositional variables. The interview was developed specifically for this study and based on an extensive review of the performance literature and the authors' own respective experiences. All the interviews were recorded for analysis.

\section{Analysis}

All recorded interviews were reviewed and analyzed by at least two authors. A descriptive qualitative analysis was conducted, supported by a web-based text analysis tool Voyant (https://voyanttools.org/). Analyses examined patterns by the four topic sections of the interview and explored differences by gender, generation, and nationality. Strategies (or patterns) were defined as highly common when reported by over half the sample, $n>10$, moderately common when reported by $n=4-9$, and unique or idiosyncratic when reported by $n=1-3$.

\section{Results}

\section{Pre-tournament routine}

In this study, we defined pre-tournament routines in terms of mental and physical preparation 1-2 weeks in advance of the event. The most common routine in this regard was a decrease in endurance training and an increase in explosivity, speed, and ghosting exercises in advance of a tournament $(n=11)$. The other routine identified as highly common was shortening the length of training sessions leading up to a tournament $(n=10)$. Some athletes reported halving the amount of their training a week in advance of the tournament, while maintaining normal training intensity, whereas others would cut down their training to $80 \%$ of the usual amount. One player remarked, "Ending sessions early builds up a hunger to play," and he felt that this hunger helped him approach his tournament matches in a more positive way. The athletes that reported reducing their training before a tournament consistently reasoned that the reduction built a hunger to play and reduced fatigue, leading to a refreshed mental and physical state.

There were several moderately common routines in tournament preparation, including an increase in the ratio of oncourt work to off-court work $(n=8)$. For example, some athletes reduced the amount of time that they spent in the gym lifting or on the bike/track doing cross-training and re-allotted some of the time to on-court work. One athlete reported, "one to two weeks before a tournament I focus on games, condition games, ghosting, fast explosive exercises, less endurance and more sharpening up on court." A good number thought tactically about an opponent $(n=7)$ and focused on match/game play in practice $(n=6)$. Some applied visualization of the venue, opponents, and tactics $(n=4)$ : With regard to visualization, one athlete discussed how in "preparing for specific tournaments, (he) would visualize the setup of the tournament, the club, the court, then first round, the second round...' and so on. And some mentioned incorporating meditation practice $(n=4)$.

There were unique routines that despite being reported infrequently seemed sensible. These included a session after an arrival flight to loosen up ( $\mathrm{n}=2)$, traveling to the tournament early to account for jetlag $(n=2)$, and doing the match warmup before practices $(n=2)$. They also included deliberately trying not to care about winning or losing in training $(n=2$ : the rationale being: "The target is the process of training, not the outcome"), thinking about specific tournament goals $(n=2)$, working with a psychologist $(n=2)$, breathing exercises $(n=1)$, video analysis of an opponent 
$(n=1)$, and scheduling practices to approximate match times $(n=1)$. A couple athletes $(n=2)$ also noted making a purposeful effort to try and be relaxed in advance of a tournament, with one athlete adding he would even have a couple beers post-practice to force that sense of normality and being relaxed.

In addition, a few athletes $(\mathrm{n}=3)$ highlighted the importance of "adaptability" -that is, fitting a physical and mental state to a situation or context. Accordingly, it is important to "pay attention to what works for one's body" and change routines to account for how one feels. "Every day you (can) feel different, so the most important thing for an athlete is to adapt." Put another way, the sport "teaches you to know yourself." This recognition has relevance in off-season training but becomes especially important during the squash season when there are many tournaments with varying breaks in between and thus many changes required based on how one feels after a particular event.

\section{Pre-match routine}

In this study, we defined pre-match routines in terms of mental and physical preparation for a specific match -with an open-ended question as to when the athletes started such routines: Their responses ranged from covering the night before through the day of the actual match. The most common component of the prematch routine included dietary regimens $(n=11)$ : This involved consistency and restrictions in both the timing and content of meals. Most athletes described what they would eat during tournaments as "simple foods," with generally less meat, especially red meat, and more carbs. Chicken and salmon were the most common sources of protein, and pasta and rice were the most common carb sources. Some athletes would carb-load in advance of their match, some throughout a tournament.

Many of the interviewed athletes reported listening to music before matches $(n=10$ : They used music in differing stages within their pre-match routine; for example, calm music on the drive to the venue, more upbeat, fast-paced music in the physical warmup, and then calm music in the cool down after a match. A number $(n=7)$ also reported adapting different types of music for different mental states that they experienced.

In addition, many reported taking a pre-match nap $(n=10$ : Of the athletes that mentioned napping, several also specified that they would be sure to wake-up at least 2-3 hours before their match time). The nap times ranged from 20 minutes to 2 hours in duration. (Because some matches would end late at night, and due to the time required for recovery and nutrition (as well as adrenaline to quiet), these athletes would fall asleep very late at night or early in the morning; as a result, they would increase their nap length to adapt for the change in their sleep pattern. One even reported skipping a morning practice session to ensure 10-11 hours of sleep).

Many engaged in a physical warmup of some kind lasting for at least 30 minutes $(n=10)$ immediately before their match. In discussing their pre-match routines, the athletes provided the most detail in their descriptions of their physical warm-ups. The most commonly included exercises in the warmup were running exercises ( $n=11$, jogging, sprinting, back-pedaling, side-shuffles, karaokes, high-knees), ghosting ( $n=11$, rehearsing squash-specific movements), and dynamic stretching ( $\mathrm{n}=10$, leg swings, lunges, side-lunges, walking-quad stretch, walking-hamstring stretch, and hip-circles). The moderately common exercises were activation exercises $(\mathrm{n}=10)$, such as air-squatting, glute bridges, and wallsits help to ready the body for the explosive movements involved in squash. A number engaged in static stretching $(n=6)$, such as a lunge stretch for the hip-flexor and touching toes to stretch hamstrings. The less commonly mentioned exercises included fast feet exercises $(n=3)$, biking $(n=2)$, hitting on court $(n=3)$, visual tracking of a moving ball $(n=1)$, and rolling $(n=2)$. A number (4) reported adapting warmup (and cool down) routines, changing them according to how their bodies felt: for example, one athlete described making warmups "longer and lighter" when tired after a previous match that was particularly draining.

Moderately common pre-match routines also included visualization the night before a match or the day of $(n=7)$ : One athlete described the visualization that he does before getting to the venue as playing the match in his head, "winning easily and how (he) needs to keep up the pace and not take (his) foot off the gas." The player also visualizes losing easily and how he would come back from a deficit, a ref who is for or against him, an opponent getting inured, and other situations so as to prepare himself for any scenario so he "doesn't panic on court. Several reported minimizing social interaction before the match $(\mathrm{n}=7)$ : Typically, these athletes would try to avoid talking to most or all people at some point before their match. Some highlighted praying $(n=4)$ : Prayer helped some athletes deal with pressure by helping to put things in perspective. One player said, prayer "makes what you're doing nothing more than fun; take the money out of the situation and it's just fun you're playing sports at the end of the day; thinking of a higher power makes what you are doing a lot more miniscule." A good number reported stopping food intake at least 2.5-3 hours before the match $(n=9)$, a morning practice hit on the day of the match $(n=7)$, reciting mantras and affirmations throughout the match day or routine $(n=6)$, a morning stretch and or roll $(n=4)$, visualization directly before the match $(n=6)$, getting advice from a coach and or family member before the match $(n=5)$, increasing the length of the physical warm-up throughout the course of the tournament $(n=4$ : the rationale being that as one progresses through a tournament the body is sorer, more fatigued, and takes longer to get activated), breathing exercises $(n=4)$, and arriving at the venue at least an hour before the match $(n=9)$.

Other unique pre-match routines included deliberately trying not to think about the match on the day before or day of a match $(n=3)$, likewise about the opponent or draw $(n=2)$. Some reported having a pre-match coffee $(n=1)$, watching videos of the opponent $(n=3)$, and a cold shower $(n=2)$. Some suggested keeping the prematch and pre-practice warmups identical $(n=2)$ : The rationale provided was that doing the same warmup with such frequency will make performing that warmup before a match an almost relaxing experience. "If you do it in practice and you've done it countless times, then doing it again but in a different setting 
could give a sense of assuredness and familiarity." One athlete also discussed how it made sense from the physical perspective: "You should warm up well before practices, and (then) you won't be tired during the match from warming up harder than you normally do. Others watched matches before playing $(n=2)$, expressed negative thoughts aloud $(n=1)$, and wrote down goals $(n=2)$. A couple reviewed their notebook $(n=2)$ : These athletes kept notebooks that included goals, affirmations, strategies for each player, analyses of matches, and other information; they would look to their notebooks to remind themselves of their affirmations and notes on their specific opponents. Others tried to preserve energy on the match day $(n=2)$ : This involved doing their best to expend as little energy as possible throughout the day of their match. One player described how she would try to not leave her hotel room as much as possible and do almost nothing all day so as to limit stimulation and preserve energy for the match.

\section{In-match routines}

In this study, we explored the application of pre-planned routines to match play and also queried about any in-match adjustment strategies. In both regards, the emphasis was on the psychological. We found no answers to be considered highly common. We did find, however, the following moderately common strategies that the athletes brought into matches: reciting cue words (mantras, affirmations or anchors) throughout the match (n=7). The words were generally positive and practical: Typical examples included "never give up," and "be aggressive," plus "volley" and "push up." A number of these athletes $(n=4)$ reported changing and adapting these words to different internal states, opponents or situations that they faced.

Some athletes also tried to accept nerves as inescapable $(n=4)$ : They discussed how they deal with pressure by spinning it in a positive light and accepting it as a reality that can't be conquered necessarily. One athlete remarked, "No nerves meant I wasn't ready." Another said, "You have to have pressure, there are a lot of things in front of you to face, so it's impossible not to have pressure. You have to find a way to put the pressure in a positive light, breathe properly, try to control it. The more you're relaxed the more focus you can get. There are ways to manage it." Another athlete suggested, "I'm so used to feeling pressure. I have been playing under it my whole life, and I have come to accept it and like it." He went on to say that he knows "something is wrong," when he goes on court and feels no pressure: "The worst thing you can do is deny the pressure, it's all about learning to play with pressure." Several of these athletes seemed to think that their level under pressure, their ability to focus in big points and still perform when feeling the nerves, was what made them elite. This thinking process appeared to be more than just an attitude; it was something some athletes would tell themselves: "That's what separates me from other people." In addition to such thinking, several athletes $(n=4)$ also reported engaging in "centering" actions to manage pressure, such as straightening racquet strings or adjusting a shoe between points. An athlete might repeat a specific centering action throughout a match, especially at moments when she felt the need to re-focus. It essentially functions as a mental reset button.
What was less frequently reported was positive thinking $(n=2)$ : A couple athletes said they made a conscious effort to think positively going up to and during a match. One also described reciting the game plan: This athlete would reread his game plan before the match and then recite it before and during the match. Another described applying breathing exercises during a game: This athlete would pay special attention to his breathing during the match itself, trying to belly breathe in between points. Some athletes tried dealing with nerves by placing the match into perspective $(n=3)$ : They dealt with pressure by contextualizing the match with regard to 'the bigger picture of life." One player said, he would remind himself "how lucky (he) was to be there, to be healthy. It's just a squash match." Another would ask herself, "If this was the last match you were ever going to play how would you want to go out?"). Others reported praying in game $(n=1)$ and trying not to think when winning $(n=1)$. Two noted thinking more when losing $(n=2)$. A few reported focusing on body language mid-game $(n=3)$ : They felt that keeping their chest upright and shoulders back did more than project confidence to the opponent, but actually improved their level. For these players, the positive body language helped them play aggressively, move better, and feel confident on court. A number described using specific cue words for certain scenarios and mental states $(n=2)$, invoking a different mantra for each opponent $(\mathrm{n}=1)$, and trying to only think in between points $(n=1)$.

\section{General disposition}

In this study, we also asked a number of questions to gain some insight into the general dispositional variables that would have some bearing on their PPRs. In this regard, the only highly common characteristic was religiosity or spirituality, having some form of faith $(n=10)$. Many of the athletes described themselves as laid back and relaxed $(n=7)$, and several mentioned meditative practice as a reflection of this disposition $(n=4)$. Several $(n=6)$ highlighted their ability to focus, especially on the big points, as something they believed set them apart. Unique characteristics included an emphasis on listening $(n=1)$ : One athlete viewed the ability to listen and intake new information and ideas from coaches, peers, and others as a vital aspect of being an elite athlete. Others again described an emphasis on adaptability $(n=3)$. These athletes discussed how important it was to be flexible and adaptable in life to be a successful athlete. As highlighted with regard to the pretournament routine, adaptability helps these athletes to listen to their bodies and minds and tailor their training and preparation to maximize their level on the day when they need to be their sharpest.

Considerations by gender, generation, and nationality. There were no differences noted by gender or by generation. However, the Egyptian and South Asian athletes uniformly reported the influence of their religious faith, whereas less than $30 \%$ of the other athletes interviewed highlighted this as a factor: This distinction by nationality was the only notable difference.

\section{Conclusions}

This study provides a foundational understanding of and insight into the preparatory routines of elite squash athletes, a unique sample of world-class performers, balanced by gender, generation, 
and nationality. The findings indicate a good deal of commonality and some individuality with regard to PPRs. They suggest the importance of the following for the athletes interviewed:

- Titrating training and emphasizing on-court training in advance of a tournament;

- Attention to dietary regimen, with emphasis on carbs and some inclusion of protein;

- Tactical work, including video analysis and strategic planning;

- Attention to sleep hygiene and use of pre-match napping;

- Dynamic physical warmup exercises (with some variety and idiosyncrasies);

- Attention to adaptability of routines and adjusting behavior to context;

- Mental work, including visualization, perspectivism, cue word use, faith-based practice, meditative practice, listening to music.

- $\quad$ Acceptance of nerves and embracing performing under pressure.

Many of the mental strategies (as well as disposition to pressure) cited above have been found to have important implications for emotion regulation and psychological resilience [11-14]. The importance of religious faith by nationality was noteworthy. Of course, the sample size limits generalizability of the findings, and the interview structure limited the nature of the information provided. Future directions will have to build on the findings to clarify which of the routines reported are most relevant for maximizing performance and how they function to promote performance $[15,16]$.

\section{References}

1. Beilock S (2010) Choke: Performing under pressure. Simon \& Schuster New York, USA.

2. Ericsson KA, Pool R (2016) Peak: Secrets from the new science of expertise. Houghton Mifflin Harcourt, New York, USA.
3. Cohn PJ (1990) Preperformance routines in sport: Theoretical support and practical applications. The Sport Psychologist 4(3): 301-312.

4. Moran AP (1996) The psychology of concentration in sports performers: A cognitive analysis. Psychology Press, New York, USA.

5. Crampton J (1989) Establishing pre-shot routines for tournament golfers: An example of the use of micro-computers in performance planning. Sports Coach 12: 9-12.

6. Cotterill S (2010) Pre-performance routines in sport: Current understanding and future directions. International Review of Sport and Exercise Psychology 3: 132-153.

7. Singer RN (2002) Pre-performance state, routines, and automaticity: what does it take to realize expertise in self-paced events? Journal of Sport \& Exercise Psychology 24(4): 359-375.

8. Brooks AW, Schroeder J, Risen JL, Gino F, Galinsky AD, et al. (2016) Don't stop believing: Rituals improve performance by decreasing anxiety. Organizational Behavior and Human Decision Processes 137: 71-85.

9. Gröpel P, Mesagno C (2017) Choking interventions in sports: A systematic review. International Review of Sport and Exercise Psychology 12(1): 176-201.

10. Perry IS, Katz YJ (2015) Pre-performance routines, accuracy in athletic performance and self-control. Athens Journal of Sports 2(3): 137-152.

11. Sarkar M, Fletcher D (2014) Psychological resilience in sport performers: A review of stressors and protective factors. Journal of Sports Sciences 32(15): 1419-1434.

12. Gross JJ (2015) The handbook of emotion regulation. ( $2^{\text {nd }}$ edn), Guilford Press, New York, USA.

13. Moore KS (2013) A systematic review on the neural effects of music on emotion regulation: Implications for music therapy practice. Journal of Music Therapy 50(3): 198-242.

14. Sobhy SK, Muran AC, Muran JC (2018) Emotion regulation and preperformance routines in competitive sports. Research \& Investigations in Sports Medicine 3: 1-3. DOI: 10.31031/RISM.2018.03.000567.

15. Mesagno C, Mullane-Grant T (2010) A comparison of different preperformance routines as possible choking interventions. Journal of Applied Sport Psychology 22(3): 343-360.

16. Morais C, Gomes AR (2019) Pre-service routines, mental toughness and performance enhancement of young tennis athletes. International Journal of Sport Psychology 50(2): 176-192.

For possible submissions Click below: 